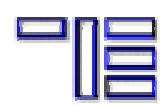

\title{
Principios justificativos de la reforma educativa: su concreción en Cataluña
}

\author{
Jaume Sarramona \\ Universitat Autónoma de Barcelona
}

Índice

1. Principios que inspiran el sistema educativo actual

1.1 Concepto de autonomía

1.2 Atención a los nuevos tiempos sociales

1.3 Cambios sociales de carácter democrático

1.3.1 Participación de la comunidad educativa

1.3.2 Igualdad de oportunidades

1.3.3 Integración de los alumnos deficientes

1.3.4 No-discriminación

2. Concepción sistémica del sistema educativo

2.1 Principios psico-pedagógicos del diseño curricular

2.2 Constructivismo

2.3 Teoría del aprendizaje significativo

2.4 Teoría del desarrollo evolutivo por etapas

2.5 Teoría del aprendizaje por uno mismo (aprender a aprender)

3. Para concluir

1. Principios que inspiran el sistema educativo actual

La Reforma vigente del sistema educativo español se inició en 1985 con la Ley Orgánica del Derecho a la Educación (LODE) y siguió en 1990 con la Ley Orgánica de Ordenación General del Sistema Educativo (LOGSE) y en 1995 con la Ley Orgánica de la Participación, la Evaluación y el Gobierno de los Centros Docentes (LOPEG).

La estructura general del sistema educativo quedó establecida del modo siguiente:

- Educación infantil, dividida en dos ciclos de 0 a 3 y de 3 a 6 años.

- Educación primaria, dividida en tres ciclos de 6 a 8 años, de 8 a 10 años y de 10 a 12 años.

- Educación secundaria obligatoria, dividida en dos ciclos, de 12 a 14 años y de 14 a 16 años. 
- Educación secundaria post-obligatoria, que abarca:

a) Ciclos de Formación Profesional de carácter medio y superior.

b) Bachillerato de dos años, con cuatro modalidades:

Tecnología, Arte, Humanidades y Ciencias Físico-Naturales.

El conjunto del sistema educativo es común para todo el Estado español, pero su estructura política en Comunidades Autónomas permite que existan diferencias de aplicación en los distintos territorios que lo componen, de acuerdo con sus necesidades, tradiciones y voluntad política de los gobiernos respectivos. Por esta razón no se puede hablar de una sola concreción de la Reforma educativa en marcha. En este artículo se presentan los principios generales que rigen para toda la Reforma y se concreta su aplicación en el territorio de Cataluña, que tiene transferidas las competencias en educación desde 1981.

Aunque en el redactado de las leyes básicas no hay explicitaciones concretas de las teorías psicopedagógicas que fundamentan la Reforma, en sus preámbulos y en los respectivos artículos, así como en otros documentos, como el Libro Blanco (1987), se pueden identificar ciertos principios teóricos básicos que justifican los cambios introducidos en el sistema educativo español. Para la concreción en Cataluña se cuenta con los documentos de desarrollo de las citadas leyes que ha editado el Departament d'Ensenyament de la Generalitat de Catalunya. De tales fuentes, más las propias reflexiones personales, surgen las explicaciones siguientes.

\subsection{Concepto de autonomía}

Concepto con diferentes niveles interpretativos y aplicativos. Empieza con la organización del Estado en Comunidades Autónomas y sigue por el grado de autonomía que se da a centros y profesores para actuar sobre el contexto inmediato.

Así se asegura que la ley tenga "un nivel de ductilidad suficiente para asegurar el marco preciso y la orientación apropiada, pero también para permitir adaptaciones y ulteriores desarrollos" (preámbulo de la LOGSE). Y se añade que a las "comunidades autónomas.... les corresponde desarrollar un papel absolutamente decisivo en la tarea de completar el diseño y asegurar la puesta en marcha efectiva de la reforma" (Ibídem). Esto se traduce en un currículum escolar con diferentes niveles de concreción. Un primer nivel, de carácter básico, está a cargo de la Administración central (estatal) más la aportación de la administración del gobierno autonómico que tiene transferidas las competencias en educación; un segundo nivel ya es responsabilidad del centro escolar y el tercero, vinculado a cada grupo concreto de alumnos, corresponde desarrollarlo a cada docente en su aula.

Ahora bien, el marco legal vigente otorga al Ministerio de Educación la exclusividad en la expedición de títulos y la determinación de las enseñanzas mínimas que justifiquen su validación para todo el territorio del Estado. 
Como ocurre en otros ámbitos de la vida política y social, existen diferentes grados de desarrollo autonómico y de conciencia de diversidad, que luego se traducen en un diferente grado de aportación de las respectivas administraciones autonómicas en sus respectivos ámbitos educativos. Buena prueba de ello es que Cataluña ya avanzó un "diseño curricular base" a mediados de los años ochenta $\mathrm{y}$, aunque muchos de sus criterios se recogieron en las directrices ministeriales, se hubiera deseado que los llamados "mínimos" para todo el Estado fuesen objetivos terminales de aprendizaje, sin especificar contenidos descriptivos ni una normativa horaria por áreas de conocimientos. De esta manera, los mínimos que determina el conjunto de la administración (primer nivel de concreción curricular) no habrían sido la suma de las directrices ministeriales más las del Departament d'Ensenyament de la Generalitat de Catalunya, sino la concreción de tales objetivos generales comunes a todo el Estado.

Otro ejemplo de esta comentada diferenciación entre Comunidades Autónomas es el hecho de que Cataluña, Euskadi y Navarra no se vincularan al retraso de dos años del calendario de aplicación de la Reforma, que el Ministerio de Educación y Cultura aprobó (1997) para el territorio que gestionaba directamente, mientras dejaba la elección de hacerlo a las Comunidades Autónomas con transferencias educativas.

En el artículo 4.2 de la LOGSE se determina que el Ministerio fijará los contenidos correspondientes al 55\% del horario escolar de las Comunidades Autónomas con lengua propia distinta del castellano; por tanto al margen de autonomía de estas administraciones autonómicas les corresponde el resto. En la práctica, no obstante, la cantidad de contenidos establecidos como mínimos por el Estado hace casi imposible tratarlos en el tiempo escolar señalado.

Si del nivel del Estado pasamos al de la Comunidad Autónoma respectiva encontraremos otros niveles de descentralización. En el caso de Cataluña, existen Delegaciones Territoriales, que ejercen las funciones de representación del Departament d'Ensenyament dentro de su territorio, y son los órganos gestores de la programación que se realiza en materia de programas educativos, de recursos personales y de los centros. Concretamente hay siete Delegaciones. Sin embargo, sus atribuciones no son siempre claras respecto a la estructura central, si bien en los últimos años ha aumentado su papel y autonomía con la jurisdicción recibida sobre el Servicio de Inspección, que también ha sido territorializado.

En Cataluña la ordenación general de la etapa de educación primaria ha dejado un margen del $10 \%$ del tiempo escolar sin atribuirlo a ninguna etapa en concreto, para así facilitar la autonomía pedagógica de los centros. En la etapa de la secundaria obligatoria, que se desarrolla íntegramente en los "Institutos de Educación Secundaria", la planificación curricular ha tomado una forma propia y de gran flexibilidad, puesto que sólo hay un $60 \%$ del tiempo lectivo que es común, mientras que el resto permite realizar a cada centro las pertinentes adaptaciones en razón del contexto y de las capacidades e intereses de sus alumnos. A tal efecto se ha adoptado el "crédito" como unidad de medida básica ( 35 horas en total), que viene a ser 
el equivalente a una asignatura trimestral de tres horas semanales. Hay que decir, no obstante, que esta estructura ha demandado recientemente ajustes y orientaciones generales para evitar la excesiva atomización de las enseñanzas, tal como ha acontecido en algunos centros. Existe la obligación de que los alumnos cursen en cada ciclo de la etapa un crédito de "síntesis", que permita la necesaria globalización de los aprendizajes adquiridos.

En el bachillerato también existe la citada organización de las materias por créditos, que permiten organizar materias anuales, especialmente las comunes y de modalidad, pero también "materias" trimestrales con las optativas. Todos los alumnos catalanes de bachillerato han de realizar un pequeño trabajo de investigación que los capacite para la recogida de información, elaboración de conclusiones y defensa de posiciones personales.

Dentro de este mismo apartado de la autonomía que comentamos se puede situar la recuperación y normalización lingüística y cultural de las comunidades con lengua propia distinta del castellano; porque ha sido gracias a ella que se ha podido avanzar en este sentido. El tema es tan importante que sin duda merece análisis más amplios que los aquí pretendidos, pero es preciso decir que la educación escolar en Cataluña tiene como objetivos generales, entre otros: "Identificar, y asumir como propias las características históricas culturales, geográficas y sociales de la sociedad catalana : tener conocimiento de los derechos de los pueblos y de los individuos a su identidad y desarrollar una actitud de interés y respecto hacia el ejercicio de este derecho".

"Comprender y producir mensajes orales y escritos con propiedad, autonomía y creatividad, en lengua catalana y castellana...."

Estos objetivos pedagógicos están amparados en las leyes educativas pero también en la Ley de Política Lingüística (1997) en la cual se dice explícitamente que una de las metas principales de la ley es el "proteger y difundir el uso del catalán entre todos los ciudadanos y ciudadanas". Los resultados son positivos en el nivel de educación primaria pero aún débiles en la secundaria. La situación actual ha de luchar contra un cierto clima de oposición, nacida en el seno de los sectores más conservadores e integristas del Estado, además de tener que compensar el peso de los medios de comunicación mayoritariamente castellano-hablantes. Los estudios realizados con los alumnos que han recibido "inmersión" lingüística en catalán confirman los resultados positivos de la misma, tanto en el dominio de las dos lenguas oficiales como en otras materias escolares.

\subsection{Atención a los nuevos tiempos sociales}

Esta realidad tiene en la "sociedad del saber" una de las expresiones que más directamente inciden sobre la educación escolar, porque la escuela se ve obligada a compartir "con otras instancias sociales la transmisión de información y de conocimientos " (preámbulo de la LOGSE), lo cual 
supone, como contrapartida, la necesidad de dar a la escuela un mayor protagonismo en "ordenarlos críticamente, para darles un sentido personal y moral...". En definitiva, se reconoce un nuevo papel a la escuela, que deja de ser monopolizadora del saber para convertirse en una institución que tiene que velar para: a) que el saber sea coherente, b) el sujeto tome posiciones personales i c) se prepare para aprovechar las diversas posibilidades informativas del medio social.

Estas tres finalidades de la escuela actual tendrán su traducción en las propuestas curriculares de aprendizaje significativo, planteando objetivos educativos vinculados con los valores y fomentando los hábitos personales de aprendizaje.

Con relación a los cambios tecnológicos de la sociedad actual y futura, la Reforma presenta como respuesta del sistema educativo la introducción de una materia concreta de Tecnología en la secundaria obligatoria y no obligatoria. Esta materia tiene como objetivo general "relacionar elementos básicos de cultura tecnológica que permiten entender los cambios que se producen en las formas de la vida: relaciones hombre-naturaleza, relaciones sociales y de producción [1].

Es sabido que la preparación en conocimientos y mentalidad tecnológica que exige la sociedad actual no puede confiarse solamente a una asignatura, aunque ésta se lo proponga de manera específica. En todo caso, la materia de Tecnología ha de tomar un cariz interdisciplinario y proyectarse más allá de sus contenidos específicos. Añádase, sin embargo, que esta proyección será difícil mientras en la formación permanente del profesorado no se haga una apuesta más clara por la dimensión tecnológica, que ha de estar presente en toda su actuación como profesional. Como es sabido, todavía se discute cuál es el perfil profesional del docente, con todo lo que esto implica respecto a la formación inicial, continua y sobre todo, en lo relativo a sus funciones específicas [2].

La introducción de los medios audiovisuales e informáticos en la escuela, como una forma más de atender el desafío de la sociedad tecnológica actual, se lleva a cabo mediante programas específicos, con resultados estimables. En el caso de Cataluña cabe citar, por ejemplo, una base de datos de los recursos existentes en este campo (Sinera) que ha sido remitida a todos los centros escolares. Actualmente, la totalidad de los centros de secundaria y un grupo importante de los de primaria están conectados a la red Internet y existe un ambicioso programa (Argor) a desarrollar en los tres próximos años sobre el uso de las nuevas tecnologías en los centros públicos.

La nueva secundaria obligatoria se propone una formación profesional "de base" para todos los alumnos, que les facilite "el logro de unas capacidades generales que, sin estar unidas a ningún campo profesional concreto, permita a los jóvenes desarrollar sus tareas profesionales según la demanda del mundo laboral [3]. Después se contemplan los llamados "programas de garantía social" para los alumnos que no superen los objetivos de la etapa, que les permitan incorporarse al mundo laboral o poder continuar estudios (art. 23.2 de la LOGSE ). 
La limitación de esta propuesta está en la concepción que se tenga de una formación profesional de base, siguiendo la tradición actual de quedar limitada a los que no puedan seguir la secundaria académica (bachillerato). Hay que insistir nuevamente en no vincularla exclusivamente a la materia de Tecnología, porque la mejor formación profesional de base que hoy se puede dar en la escuela es el dominio del lenguaje, el desarrollo del pensamiento matemático y el hábito de aprender, así como la adquisición de los hábitos de comportamiento que exige la actividad laboral: trabajo en colaboración, disciplina, responsabilidad, etc.

Cataluña ha adoptado un modelo específico para la organización de los programas de garantía social. Se realizan en acción coordinada entre los Departamentos de Enseñanza, Trabajo, Bienestar Social Administraciones locales y la iniciativa privada, a fin de integrar y aprovechar toda la oferta de formación no reglada que ya existe, en especial los programas de formación ocupacional, además de los más específicos de "transición al trabajo". De los 5.119 alumnos potenciales de tales programas en el curso 1997-98, $2.878 \mathrm{se}$ inscribieron en los mismos.

Situados ya en la formación profesional específica, que aparece en la secundaria no obligatoria, se contempla que una parte de la formación se realice en los centros de trabajo. La Ley ha querido prestigiar la formación profesional frente al bachillerato académico, pidiendo para el acceso a ambos la posesión del título de graduado de secundaria. El futuro dirá si ha sido una buena opción, dadas las limitaciones laborales del mercado, así como el costo de las especialidades de F.P. diseñadas. Y es que la formación profesional ha de vencer la tradición de considerarla una salida de segundo nivel, dirigida a los alumnos menos capacitados; buena prueba de ello es que en el presente aproximadamente el $70 \%$ de los alumnos que obtienen el título de la secundaria obligatoria siguen estudios de bachillerato.

Los estudios de formación profesional tienen en las practicas laborales uno de sus puntos más críticos. Hay que decir que en Cataluña existe una buena experiencia de vinculación entre la formación profesional reglada y las prácticas en las empresas, mediante el "Programa Escuela-Trabajo", que permitió atender a 29.700 alumnos el curso 96-97. Esto sin olvidar la creación en el año 86 del llamado "Instituto Catalán de Nuevas Profesiones" (INCANOP), que ha trabajado desde entonces en el desarrollo de nuevas profesiones y en los consiguientes planes de formación, además de participar en multitud de proyectos europeos.

El Departament d'Ensenyament de Cataluña tiene un "Programa de Información y de Orientación al estudiante", que ofrece ayudas a los jóvenes para decidir qué estudios profesionales y universitarios les pueden ser más adecuados. En esta misma línea se puede situar la celebración anual en el marco de la Feria de Muestras de un "Salón de la Enseñanza", para mostrar toda la oferta educativa formal e informal del país, que en el caso de la formación profesional de grado medio y superior está recogida en el correspondiente "mapa", hecho público en 1998. 


\subsection{Cambios sociales de carácter democrático}

La LOGSE significó la renovación del conjunto del sistema educativo no universitario, completándose así el marco legal que substituyó a la Ley General de Educación de 1970. Se trata de una ley que ha de preparar las futuras generaciones para vivir en democracia, no solamente en los límites estatales, sino europeos y mundiales. Las características esenciales de una sociedad democrática son diversas, pero destacaremos aquéllas que son explícitamente señaladas en el texto de la ley.

\subsubsection{Participación de la comunidad educativa}

Ya la ley de 1985 (LODE) estructuró la participación de los agentes sociales en el sistema educativo y actualmente se rige por la Ley Orgánica de la Participación, la Evaluación y el Gobierno de los Centros Docentes de 1995, determinando que en todos los centros públicos y privados concertados se creará un Consejo Escolar de Centro, con atribuciones de órgano de participación y de gobierno. Entre otras atribuciones tiene las siguientes:

- Aprobar el plan general del centro

- Aprobar el proyecto educativo del centro

- Elegir al director/a

- Aprobar el plan de actividades extraescolares y complementarias

- Participar en la evaluación externa del centro

- Y en el caso de los centros privados concertados, participar en el nombramiento y cese del profesorado.

Estos Consejos están integrados por profesores, padres, alumnos, personal no docente y un representante municipal. En los centros concertados no figura este último y la titularidad cuenta con la presencia de tres miembros.

Existen Consejos Escolares Municipales y Territoriales, Consejo Escolar Autonómico y del Estado. Estos últimos dictaminan la normativa legal, aunque sus informes no son vinculantes, mientras que los municipales y territoriales sólo tienen responsabilidades de participación.

Con respecto al funcionamiento práctico de los Consejos se puede asegurar que se ha avanzado mucho, pero siendo la experiencia positiva, queda aún camino por recorrer, en especial respecto al papel de los padres, cuya participación en el momento de elegir a sus representantes es muy baja, puesto que oscila entre un $16 \%$ de votantes en primaria al $6 \%$ en secundaria. Con respecto a los Consejos Escolares de las respectivas Comunidades Autónomas y del Estado se puede citar la interesante experiencia de la realización de unas Jornadas Anuales donde participan todos ellos para analizar un tema de interés común; las de 1998 se refirieron a la secundaria obligatoria y sus conclusiones fueron hechas públicas y remitidas a las administraciones respectivas. 


\subsubsection{Igualdad de oportunidades}

Una primera forma de entender la igualdad de oportunidades de todos los alumnos en el sistema educativo ha sido la extensión de la enseñanza omún obligatoria hasta los 16 años. Esto ha llevado implícita la aplicación del principio de la "comprensividad", entendida como una escolarización común pero con metodología diversificada y optatividad de materias. Este criterio queda recogido en el texto de la LOGSE cuando se fija, en la secundaria obligatoria por ejemplo, que la "metodología didáctica... se adaptará a las características de cada alumno" (art. 20.4); así mismo se dice en primaria que "la metodología didáctica se orientará al desarrollo general del alumno... y que la enseñanza tendrá un carácter personal y se adaptará a los diferentes ritmos de aprendizaje de cada niño" (art. 14.3).

La solución no es sencilla y los resultados posteriores a la etapa de la enseñanza no están garantizados para todos los casos. Existen razones de tipo social y pedagógico para procurar la comprensividad, siempre que se cuente con un alto nivel de profesionalidad por parte del profesorado, especialmente de secundaria, que apliquen metodologías y técnicas diversificadas; lo cual contrasta con la práctica realizada hasta ahora, básicamente selectiva.

En estos momentos, al indudable costo que supone la comprensividad tal como se ha indicado, se une la resistencia que algunos sectores docentes y familiares plantean, bajo el temor de que suponga un perjuicio para los alumnos más aventajados. Ello explica que exista la tendencia a definir "itinerarios" cerrados para los grupos de alumnos que ya en la secundaria obligatoria se quieren canalizar hacia el bachillerato, la formación profesional o directamente al mundo laboral, tal como se ha establecido con carácter general en el territorio de Navarra.

En aras de la igualdad de oportunidades, también se ha determinado:

- La regularización de la etapa pre-obligatoria.

- Una plaza escolar gratuita en los niveles obligatorios

- Una política de becas para la secundaria post-obligatoria.

- Unas posibilidades de educación compensatoria para los adultos.

- Un curriculum básico común para todos.

Estas medidas serán efectivas siempre que la oferta de plazas públicas desde los primeros años - sea amplia y de calidad; en caso contrario, las diferencias podrían venir dadas por el tipo de escuela a la que se asiste, aunque la enseñanza sea teóricamente la misma para todos. En el caso de Cataluña, la necesidad de atender carencias históricas en la enseñanza obligatoria y la elevada apuesta por aplicar la Reforma en su integridad, no ha permitido hasta ahora dedicar más recursos a crear plazas públicas para los primeros años, de manera que este nivel educativo está mayoritariamente cubierto por el sector privado y municipal. La proporción del sector público y privado en la secundaria obligatoria de Cataluña es, respectivamente del 
59 y del 41\% (curso 1997-98).

La igualdad de oportunidades, unida a los cambios sociales acelerados, justifica la formación permanente de los adultos, facilitándoles el acceso a las titulaciones académicas a partir del mismo ámbito laboral y sin requisitos académicos previos. Véanse las siguientes medidas que prevé la ley:

a) Obtención del Graduado en Secundaria para mayores de 18 años mediante pruebas específicas

b) Aumento de la oferta de enseñanza a distancia

c) Obtención del título de bachillerato para mayores de 23 años

d) Acceso a la Universidad para mayores de 25 años

e) Convalidación entre la F.P. de grado medio y superior

mediante pruebas específicas para los mayores de 21 años

La LOGSE considera la educación de adultos integrada en el sistema general. Sin embargo, en Cataluña existe una ley específica para la educación de adultos, aprobada en 1991. El hecho de tener una ley propia, y que la educación de adultos dependa de la Consejería de Bienestar Social desde 1988, ha permitido darle un tratamiento específico, aunque a costa de una cierta disociación con la enseñanza convencional. Actualmente está realizado el "mapa de la educación de adultos" y se cuenta con programas específicos, como el de preparación para la obtención del título de Graduado Escolar por televisión (Graduïs, ara pot) además de la enseñanza convencional dirigida a más de treinta mil personas en los centros presenciales.

Los adultos también pueden seguir a distancia los estudios de la actual secundaria en el "Instituto Catalán de Enseñanza Secundaria a Distancia" (ICESD), que depende del Departament d'Ensenyament y que recoge la estructura catalana del desaparecido "Instituto Nacional de Bachillerato a Distancia", de ámbito estatal. En el futuro este centro impartirá a distancia la enseñanza secundaria obligatoria y no obligatoria.

La continuidad de la enseñanza a distancia propia de Cataluña culminó el curso 95-96 en el nivel universitario, gracias a la creación de la "Universitat Oberta de Catalunya" (UOC) que supone una alternativa al monopolio de la "Universidad Nacional de Educación a Distancia" (UNED), directamente vinculada al Ministerio de Educación y de cobertura estatal, sin atención a la diversidad cultural y lingüística de los diversos territorios del Estado. Otras universidades catalanas se han iniciado también en la oferta de algunos estudios a distancia.

La enseñanza a distancia, admitida ya en todo el mundo como una alternativa válida para la educación de adultos, aún no tienen en el sector público catalán el relieve que le corresponde. Se parte de antecedentes de centralización pero parece que en las autoridades aún no hay convicción clara de sus posibilidades, además de tener que romper rutinas e intereses corporativos de los educadores de adultos y del profesorado de cursos nocturnos. En el sector privado, en cambio, la enseñanza a distancia tiene una fuerte implantación en educación no reglada. 


\subsubsection{Integración de los alumnos deficientes}

Siguiendo las medidas ya aplicadas hace unos años en Cataluña, la LOGSE consolida el principio de la integración escolar de los "alumnos con dificultades educativas especiales". Con esta finalidad, se prodigará la creación de equipos psico-pedagógicos que proporcionen las ayudas complementarias pertinentes (art. 37).

La materialización adecuada de esta propuesta sin duda ha de facilitar la incorporación social posterior de estos alumnos deficientes, siempre que se llegue a disponer en los centros ordinarios de los recursos humanos y técnicos necesarios para desarrollar todas sus posibilidades educativas. En caso contrario se pueden ver más marginados que en los centros de educación especial. El debate sobre la eficacia de las medidas de integración de los niños deficientes en la escuela ordinaria aún no está cerrado, pero el proceso continúa de manera progresiva, hasta el punto de que actualmente, a raíz de la ley de 1995, se contempla que esta integración afecte con carácter obligatorio a todos los centros sostenidos con fondos públicos (públicos y privados concertados).

Cataluña tiene organizados desde hace años los llamados "equipos de atención psicopedagógica" (EAPs), de ámbito comarcal, donde hay profesionales especialistas en atención a deficientes y en orientación escolar, que colaboran con el profesorado de los centros para atender a los alumnos necesitados. Estos equipos se complementan con la presencia de especialistas en los centros de secundaria. Estos especialistas atenderán a los alumnos con dificultades, además de colaborar con el profesorado para materializar la tutoría personal. Los recursos disponibles pueden ser una gran limitación para poder contar con todos los especialistas en los centros. Destaquemos que hay "centros de recursos" para atender a los deficientes auditivos y EAPs específicos para otros tipos de deficientes.

\subsubsection{No-discriminación}

Sobre estos aspectos las leyes educativas (LOGSE) recogen los principios de la Constitución y se cita explícitamente la no discriminación en función del sexo (art. 2.3c), "superando los estereotipos sociales asimilados a la diferenciación por sexo, empezando por la propia construcción y uso del lenguaje" (preámbulo) y siguiendo por la elaboración de los materiales didácticos en general (art. 53.3). En este ámbito se ha avanzado considerablemente, si bien se deberá persistir en el tema.

Lo que ahora constituye un nuevo desafío, como en el resto de los países europeos, es la atención a las diversas culturas que confluyen en un mismo territorio, como consecuencia de los movimientos migratorios. Este es un tema de especial preocupación en Cataluña, tierra acogedora de inmigraciones desde siempre, y que ahora ha de hacer compatible el respeto a la diversidad con al integración social de los inmigrantes y la recuperación de la propia identidad cultural. 
Desde hace años existe un programa denominado de "educación compensatoria" pero, tras un estudio encargado a un equipo de profesores de la Universidad Autónoma de Barcelona y la elaboración de un "mapa escolar intercultural de Cataluña" (1994) se ha podido conocer el alcance de la situación[4] . Como consecuencia de este estudio se prepararon unas orientaciones específicas sobre el tratamiento de la interculturalidad, incorporándolas a los curriculo de secundaria, y se ha determinado este ámbito como objetivo de tratamiento preferente.

Paralelamente, a través del Programa de Educación Compensatoria, se siguen realizando actividades de formación del profesorado y dando subvenciones a entidades que atienden a alumnos de culturas minoritarias. De manera concreta se puede citar que el curso 96-97, 3.330 alumnos recibieron ayuda directa del programa, unos 240 centros recibieron atención sistemática semanal y otros 260 la recibieron más puntualmente.

\section{Concepción sistemática del sistema educativo}

Desde este punto de vista se puede justificar la introducción de la evaluación permanente el sistema educativo, como una garantía de la adaptación a las exigencias que en cada momento se plantean (art. 62 de la LOGSE). Por ello se creó en Madrid el "Instituto Nacional de Calidad y Evaluación" (INCE), en el cual "participaran las Comunidades Autónomas", pero se especifica también que las "administraciones educativas evaluarán el sistema en el ámbito de sus competencias" (art. 62.2), de ahí que se creara en Cataluña el "Consejo Superior de Evaluación del Sistema Educativo" (1994). Sin embargo, la delimitación de las atribuciones entre el organismo estatal y el de las Comunidades Autónomas no está muy claro, y puede ser un foco de debate permanente. Como ejemplo se puede citar el estudio realizado sobre los resultados escolares de los alumnos de 14 y 16 años que el INCE realizó durante el curso 1996-97, en el cual no participaron Andalucía y Canarias, y cuyos resultados fueron objeto de profunda controversia al hacerse públicos bajo la fórmula de comparación entre territorios [5].

Indudablemente es necesario una evaluación del sistema. La LOGSE no previó una evaluación concreta de los centros y de los profesores pero la ley de 1995, denominada precisamente "Ley Orgánica de la Participación, la Evaluación y el Gobierno de los Centros Docentes" contempla de manera explícita la obligación de evaluar los centros, en la cual se señala que "participarán los Consejos Escolares de los centros".

La materialización de esta evaluación se ha iniciado en Cataluña (1998) mediante un programa que contempla la doble perspectiva de la evaluación interna y externa para todos los centros de primaria y los de secundaria que ya han completado la Reforma. La primera la llevan a cabo los propios centros, mediante unas directrices y materiales facilitados al efecto, y la evaluación externa la ejecutan los inspectores. El programa tiene una fase inicial prevista de dos años de duración. 
Respecto al tema de la estructura interna de los centros, hasta el año 1996 siguió la concepción de los equipos directivos no profesionalizados, esto es, designados por los Consejos Escolares de los centros respectivos sin más requisito que ser docentes del mismo centro, de acuerdo con las directrices señaladas en la Ley Orgánica del Derecho a la Educación (LODE) de 1985. Pero la insatisfacción general del sistema, que llevó a que la misma administración tuviera que nombrar directamente a una gran parte de los directores, provocó que en la citada ley de 1995 se introdujeran medidas potenciadoras de la función directiva, que tienden a una cierta profesionalización, como la exigencia de una cierta preparación previa y la consolidación del correspondiente complemento económico, pero sin incluir una clara tarea de supervisión interna.

Cataluña hasta ahora se ha preocupado de la formación de los directores escolares una vez eran nombrados. Formación circunscrita a un cierto territorio y con efectos muy limitados, ya que la dirección se ejercía también por un período limitado, y en pocos años se producía prácticamente una renovación total de los cargos. De estos antecedentes resulta positiva la creación de las llamadas "Juntas de Directores", con las cuales la administración catalana puede establecer diálogo y mantener un contacto permanente de cariz institucional.

Algo parecido se podría decir de los inspectores, ya que el Cuerpo de Inspectores fue suprimido en $1985 \mathrm{y}$ ha sido legalmente restablecido diez años después. Durante este período se accedió a la "función" inspectora sin oposición y sin requisitos previos de preparación, aunque se ofertara luego a los designados; este sistema ha sido motivo de fuertes controversias.

Cataluña tiene actualmente un Plan Director para la Inspección para garantizar una preparación técnica adecuada, dentro del cual destaca la iniciativa de un Master en Supervisión y Evaluación de la Educación, dirigido a los inspectores en activo, iniciativa única en todo el Estado, del cual se han realizado tres ediciones que han permitido participar a un total de unos 120 inspectores en total.

Un sistema dinámico no solamente ha de tener "feed-back" sino, como se dice en lenguaje cibernético, ha de tener "feed-before", es decir, proyección prospectiva. Aquí entra entonces la innovación y la investigación. La ley dice que "las administraciones educativas fomentarán la investigación y favorecerán la elaboración de proyectos que incluyan innovaciones curriculares, metodológicas, tecnológicas, didácticas y de organización de los centros docentes" (art. 59); si bien se matiza que las experimentaciones que afecten a la ordenación general del sistema educativo necesitarán autorización expresa del gobierno (art. 59.2). La verdad es que aún hay mucho terreno por recorrer en la vinculación entre la investigación básica y su aplicación a los centros.

La innovación educativa tiene una fuerte tradición en Cataluña, que arranca de principios de siglo, aunque pasara un paréntesis durante la dictadura franquista. Con motivo de la Reforma educativa, se fomentó la innovación a través de la red de "centros experimentales" que la anticiparon, si bien las 
experiencias han sido de calidad diversa. Asimismo se ha instaurado la concesión de licencias pagadas por un año a maestros funcionarios para poder realizar proyectos educativos innovadores.

En 1995, por encargo de la Dirección General de Universidades y del mismo Departamento de Enseñanza, se realizó un estudio sobre la situación de la investigación educativa en Cataluña en los últimos 10 años [6]. A partir de los datos obtenidos se han podido determinar las líneas que es preciso seguir en el futuro, como consecuencia de la integración europea y de la aplicación de la Reforma. Pero aún se carece de un plan general de investigación educativa, que canalice y coordine todos los esfuerzos realizados por instancias diversas.

Tanto la evaluación como la innovación han de conducir a la mejora de la calidad del sistema a que hace referencia todo el título cuarto de la LOGSE. A los citados elementos hay que añadir todo lo vinculado con la formación permanente del profesorado. Esta se presenta como un derecho y un deber de los profesores, así como una responsabilidad por parte de las administraciones educativas y de los propios centros (art. 56.2).

La formación permanente del profesorado también tiene una gran tradición en Cataluña, hasta el punto de que sus "escuelas de verano", iniciado en 1914 y recuperado en plena época franquista (1966), han servido de modelo en todo el Estado. Actualmente hay multitud de iniciativas de formación, además de un Plan General de Formación organizado por el Departament d'Ensenyament desde 1989 y renovable cada cuatro años. Los principales esfuerzos se han centrado en la aplicación de la Reforma.

La Ley no hace ninguna referencia al posible reconocimiento de méritos al profesorado en función de la eficacia profesional demostrada, pero el Ministerio llegó en su día a un acuerdo con los sindicatos para reconocer como mérito la acreditación de un cierto número de horas de formación (100) cada período de seis años (sexenios). En Cataluña también existe un criterio de promoción retributiva en la misma línea del Ministerio, si bien contempla otros aspectos además de la formación. El hecho fáctico es que no existe en todo el Estado una auténtica "carrera docente" que estimule la calidad (controlada) del trabajo en las aulas.

Para completar el conjunto de medidas referidas a la calidad del sistema aún será necesario citar los recursos y la metodología educativa. Cataluña tiene una red de "centros de recursos", algunos de ellos especializados, que actúan sobre un territorio concreto, facilitando información y formación en su ámbito, además de ser dinamizadores de innovación pedagógica. La situación económica en el momento de redactar la ley era bastante más optimista de la actual, de manera que la práctica limita sensiblemente estos propósitos legales.

\subsection{Principios psico-pedagógicos del diseño curricular}

Como era lógico esperar, en unas leyes básicas no aparecen aspectos 
técnicos concretos de fundamentación curricular ni su aplicación; no obstante sí aparece una definición de curriculum en el artículo cuarto de la LOGSE, "a los efectos de lo que se dispone la ley", y que "entiende por curriculum el conjunto de objetivos, contenidos, métodos pedagógicos y criterios de evaluación de cada uno de los niveles, etapas, ciclos, grados y modalidades del sistema educativo que orienta la práctica docente ". Se opta, pues, por un diseño curricular con objetivos y contenidos, métodos y criterios de evaluación, para así poder señalar los mínimos obligatorios para todo el Estado en todos estos apartados.

\subsection{Constructivismo}

"Los principios psico-pedagógicos que soportan el diseño curricular base que se presenta se sitúan en una concepción constructivista del aprendizaje escolar y de la intervención pedagógica entendida en sentido amplio " (Libro Blanco, p.31).

Como se señala en los preámbulos de los textos correspondientes a los currículos de las áreas de la secundaria obligatoria que edita el Departament d'Ensenyament de Cataluña "una concepción constructivista del aprendizaje considera que todo conocimiento es construido por la persona cuando interacciona con el medio y trata de comprenderlo". Esto exige la aplicación de diversas estrategias docentes, siempre bajo el común denominador de captar que el objeto fundamental del aprendizaje escolar es la construcción del conocimiento del alumno. Solamente comentar que esta teoría solicita un nivel de conocimiento psicopedagógico por parte del profesorado que supere las simples palabras y directrices externas, ya que supone dominar de manera aplicativa teorías concretas de aprendizaje (Bruner, Vigotski, Piaget....) y esto no se da siempre.

\subsection{Teoría del aprendizaje significativo}

"El proceso de enseñanza y aprendizaje puede dar lugar tanto a aprendizajes significativos como a aprendizajes repetitivos. Si el nuevo material de aprendizaje se relaciona de forma significativa y no arbitraria con lo que el alumno ya sabe, puede llegar a asimilarse e integrarse en una estructura cognitiva previa, produciéndose entonces un aprendizaje significativo capaz de cambiar esta estructura previa, al tiempo que resulta sólido y duradero (Libro Blanco, p.32).

Este texto justificativo de las bases del diseño curricular remite directamente a teorías cognitivas como la de Ausubel, que en contraste con la teoría del aprendizaje por descubrimiento de Bruner, propone una metodología de cariz deductivo ("enseñanza expositiva"), donde los profesores tienen que presentar a los alumnos materiales altamente estructurados $y$ secuencializados, que se vinculen con sus estructuras cognitivas previas.

En los textos curriculares catalanes se insiste en que el aprendizaje no se interioriza si no es significativo, es decir "si el alumno no puede relacionar la 
nueva información o campo de experiencias que se el ofrece con algún elemento de su estructura cognitiva"...."Un aprendizaje significativo es a la vez un aprendizaje funcional, ya que el alumno ha de ser capaz de aplicarlo de manera efectiva".

\subsection{Teoría del desarrollo evolutivo por etapas}

"La psicología genética ha puesto de relieve la existencia de una serie de períodos evolutivos con características cualitativamente diferentes entre sí, que condicionan en parte los posibles efectos de las experiencias educativas escolares sobre el desarrollo del alumno. La intervención educativa tiene, pues, que partir de las posibilidades de razonamiento y de aprendizaje que las capacidades que caracterizan estos estadios dan al alumno" (Libro Blanco, p.32).

No se cita una teoría concreta del desarrollo pero queda claro que se defiende la concepción de las etapas evolutivas como condicionador de las capacidades de aprendizaje de los alumnos, sirviendo así el desarrollo evolutivo como base, a la vez que límite de la intervención educativa y de las mismas teorías constructivistas. El texto citado cita explícitamente las teorías piagetianas. Se dice también que "aprender significativamente supone modificar los esquemas de conocimiento que tiene el alumno... Durante el proceso de aprendizaje el alumno debería recibir una información que entre el alguna contradicción con los conocimientos que posee hasta entonces, y de esta manera rompa el equilibrio inicial de sus esquemas de conocimiento" ( p. p. 33-34).

En Cataluña ha habido siempre una gran sensibilidad por los principios pedagógicos piagetianos, como lo demuestran las experiencias de pedagogía operatoria realizadas por el Instituto Municipal de Educación de Barcelona, y los estudios realizados a tal efecto en las universidades catalanas.

\subsection{Teoría del aprendizaje por uno mismo ( aprender a aprender)}

"La intervención educativa debe tener como objetivo prioritario el posibilitar que los alumnos realicen aprendizajes significativos por sí solos, es decir, que sean capaces de aprender a aprender. Por lo tanto, hay que prestar especial atención a la adquisición de estrategias cognitivas de planificación y regulación de la propia actividad de aprendizaje". (Libro Blanco, p.33).

El principio del "aprender a aprender" implica tomar conciencia de los procesos mediante los cuales se aprende, esto es, de los procesos metacognitivos. Tales procesos ya habían sido referidos por Vigotsky pero han sido autores contemporáneos quienes les han dado especial relieve, concretamente los vinculados a las teorías cognitivas del aprendizaje, al proponer técnicas concretas de cómo llevarlos a cabo. Un buen ejemplo es Novak (1988), con la elaboración de los "mapas conceptuales". 
La perspectiva metacognitiva no trata de desarrollar exclusivamente estructuras cognitivas al margen de los conocimientos, sino que se propone lograrlas al tiempo que se adquieren los mismos conocimientos necesarios para la culturalización y preparación académica. La cuestión está en adquirir conocimientos tomando conciencia del modo cómo se logran. El "cómo" se aprende se vincula estrechamente el "qué" se aprende.

\section{Para concluir}

Sin duda un sistema educativo ha de estar en permanente reforma para adaptarse a las exigencias de cada país y momento histórico, y de esta exigencia permanente siempre surgen deficiencias.

La generalización de la Reforma del sistema educativo español, con las diversas variantes que pueda adoptar en las diversas Comunidades Autónomas que lo gestionarán directamente, ha coincidido con unos tiempos de ajuste económico por exigencias de la unión monetaria europea lo que ha ocasionado dificultades para su implantación en toda la amplitud prevista la cual, como se ha comentado, resulta de elevado costo económico. Pero esto no es todo.

El cambio de gobierno acontecido en 1996, con la entrada del Partido Popular que en su día no votó la LOGSE ha planteado interrogantes sobre el futuro mismo de la Reforma, sobre la cual ha manifestado sus reticencias en diversas ocasiones. El cambio no se ha producido, a excepción del ya citado respecto al calendario de aplicación, por carecer de la mayoría parlamentaria necesaria pero se advierten claros indicios del deseo de llevarlo a cabo por vías indirectas [7], para lo cual cuenta con el apoyo de los sectores más conservadores del profesorado de secundaria.

No se trata de mitificar la Reforma, por supuesto, la cual puede y debe ser reformada a su vez si se advierte claramente su necesidad. Pero los esfuerzos aplicados por el conjunto de la comunidad educativa en los últimos años para modernizar las estructuras y la acción pedagógica merece de un cierto período de tranquilidad y de confianza en el camino emprendido. Luego, realizadas las correspondientes evaluaciones, será el momento de ejecutar los ajustes pertinentes.

\section{NOTAS}

[1] Departament d'Ensenyament : Tecnología, Barcelona, 1993, p.41

[2] En este sentido se puede destacar la aportación hecha por el "Consell Escolar de Catalunya", que ha elaborado (1993) un documento sobre "deontología docente", y otro sobre "profesionalidad en educación" (1995).

[3] Introducción común a diversos documentos del Departament d'Ensenyament sobre los currículos de secundaria. 
[4] Sarramona, J (dir) y otros: L'educació intercultural a Catalunya, Direcció General d'Ordenació Educativa, Departament d'Ensenyament, doc. pol., 1994.

[5] El conjunto de los resultados ha sido publicado con el título Diagnóstico General del Sistema Educativo, INCE, Madrid, 1998. El Instituto Superior de Evaluación de Cataluña ha realizado un análisis de detallado de los datos resultantes de los alumnos catalanes en el estudio titulado: Resultats acadèmics de l'alumnat de 14 i 16 anys a Catalunya, Consell Superior d'Avaluació, Departament d'Ensenyament, 1998.

[6] Llibre Blanc de la Recerca Educativa a Catalunya (1986-95), investigación dirigida por Sarramona, J. . Publicación en soporte electrónico, Departament d'Ensenyament, 1998.

[7] El ejemplo paradigmático fue la propuesta de reforma de la enseñanza de las Humanidades en la educación secundaria que, sin contar con resultados fiables sobre los aprendizajes logrados en este nivel educativo, pretendía modificar y ampliar los contenidos curriculares mínimos en varias materias además de introducir propuestas que afectaban a la misma metodología pedagógica y a la evaluación. Tras un fuerte enfrentamiento político con toda la oposición, en especial con los gobiernos autonómicos de Andalucía, Canarias, Cataluña y Euskadi, el tema se saldó con la creación de un grupo de trabajo que presentó en junio de 1998 unas reflexiones sobre el tema (Dictamen) mucho más matizadas que las deseadas por el Ministerio de Educación y Cultura, gracias a la presencia de representantes de las diversas Comunidades Autónomas. Sobre este tema véase: Sarramona, J.: "La pretesa reforma de les Humanitats", Educar, $\mathrm{n}^{\circ}$ 24, Universitat Autònoma de Barcelona (en prensa).

(C) Ediciones Universidad de Salamanca.

Páginas mantenidas por Francisco Ignacio Revuelta Domínguez

y Luis González Rodero

webmrte@usal.es 